\title{
Ground States of Nonlinear Schrödinger-Kirchhoff Type Equation
}

\author{
Weidan Li \\ College of Science, University of Shanghai for Science and Technology, Shanghai, China \\ Email: dorallll@163.com
}

How to cite this paper: Li, W.D. (2020) Ground States of Nonlinear SchrödingerKirchhoff Type Equation. Journal of $A p$ plied Mathematics and Physics, 8, 307-314. https://doi.org/10.4236/jamp.2020.82025

Received: January 10, 2020

Accepted: February 10, 2020

Published: February 13, 2020

Copyright (C) 2020 by author(s) and Scientific Research Publishing Inc. This work is licensed under the Creative Commons Attribution International License (CC BY 4.0).

http://creativecommons.org/licenses/by/4.0/

\begin{abstract}
In this paper, we considered with the following Schrödinger-Kirchhoff type problem: $-\left(a+b \int_{R^{N}}|\nabla u|^{2} \mathrm{~d} x\right) \Delta u+V(x) u=f(x, u)$ in $R^{N}$. We put forward general assumptions on the nonlinearity $f$ with the subcritical growth and we find a ground state solution being a minimizer of the energy functional associated with a Nehari-Pankov manifold by using a linking theorem.
\end{abstract}

\section{Keywords}

Schrödinger-Kirchhoff, Linking, Ceramisequence, Fatou's Lemma

\section{Introduction}

Consider the following nonlinear Schrödinger-Kirchhoff type problem:

$$
-\left(a+b \int_{R^{N}}|\nabla u|^{2} \mathrm{~d} x\right) \Delta u+V(x) u=f(x, u) \text { in } R^{N}
$$

where constants $a>0, b \geq 0, V \in\left(R^{N}, R\right)$ and $f \in\left(R^{N} \times R, R\right)$ satisfy some assumptions.

In (1.1), if $a=1, b=0$, then Equation (1.1) is the following well-known Schrödinger equation:

$$
-\Delta u+V(x) u=f(x, u), \text { in } R^{N}
$$

The Schrödinger equation has been studied by Brezis and Lieb [1] for a general class of autonomous, and by many authors [2] [3] [4] for periodic data.

If $V(x) \equiv 0$ and $R^{N}$ are replaced by a smooth bounded domain $\Omega \in R^{N}$, then Equation (1.1) is a Dirichlet problem of Kirchhoff type [5]:

$$
\begin{cases}-\left(a+b \int_{R^{N}}|\nabla u|^{2} \mathrm{~d} x\right) \Delta u=f(x, u) & \text { on } \Omega \\ u=0, & \text { in } \partial \Omega\end{cases}
$$


Many interesting studies by variational methods can be found in [6]-[11]. It is related to the following stationary analogue of the equation:

$$
u_{t t}-\left(a+b \int_{R^{N}}|\nabla u|^{2} \mathrm{~d} x\right) \Delta u=f(x, u)
$$

which is an extension of classical D'Alembert's wave equation for free vibrations of elastic strings. Kirchhoff's model takes into account the changes in length of the string produced by transverse vibrations. Some early studies of Kirchhoff equation can be refer to in [12]-[19] and the references therein.

In order to reduce some extra statement we need to describe the eigenvalue of the Schrodinger operator $-a \Delta+V$. We are considered the sequence $\left\{\lambda_{i}\right\}$, $\lambda_{i}<0<\lambda_{i+1}<\lambda_{\infty}=\lim _{i \rightarrow \infty} \lambda_{i}$ of minimax values, it is known that $\lambda_{\infty}$, if finite, is lower bound of the essential spectrum $\sigma(-a \Delta+V)$ of Schrodinger operator $-a \Delta+V \quad$ (many details can see [20]).

Our aim is to study ground state solutions to (1.1) with a class of nonlinearities. The energy functional $I: X \rightarrow R$ given by:

$$
I(u)=\frac{a}{2} \int_{R^{N}}|\nabla u|^{2} \mathrm{~d} x+\frac{b}{4}\left(\int_{R^{N}}|\nabla u|^{2} \mathrm{~d} x\right)^{2}+\frac{1}{2} \int_{R^{N}} V(x) u^{2} \mathrm{~d} x-\int_{R^{N}} F(x, u) \mathrm{d} x
$$

Set $F(x, u)=\int_{0}^{u} f(x, s) \mathrm{d} s$. We make the following assumptions:

(V) $V \in C\left(R^{N}\right)$ bounded from below, there is $M>0$, such that

$$
\operatorname{meas}\left\{x \in R^{N} \mid V(x)<M\right\}<\infty
$$

(F) $f: R^{N} \times R \rightarrow R$ is measurable in $x \in R^{N}$ and continuous in $u \in R$ for a.e. $x \in R^{N}$.

$F: R^{N} \times R \rightarrow R$ is differentiable with respect to the second variable $u \in R$ and $F(x, 0)=0$ for a.e. $x \in R^{N}$.

(F1) There are $A>0$ and $2<p<2^{*}=\frac{2 N}{N-2}$, such that

$$
|f(x, u)| \leq A\left(1+|u|^{p-1}\right) \text { for all } u \in R \text { and a.e. } x \in R^{N} \text {. }
$$

(F2) $f(x, u)=o(|u|)$ as $|u| \rightarrow 0$ uniformly in $x \in R^{N}$.

Our goal is to find a ground state solution of Equation (1.1) i.e., a critical point being a minimizer of $I$ on the Nehari-Pankov manifold defined as follows:

$$
\mathcal{N}=\left\{u \in X \backslash X^{-} \mid\left\langle I^{\prime}(u), u\right\rangle=0 \text { and }\left\langle I^{\prime}(u), v\right\rangle=0 \text { for any } v \in X^{-}\right\} .
$$

Since $\mathcal{N}$ contains all critical points of $I$, then a ground state is a energy solution. To conquer the linking geometry of $I$ and to structure $\mathcal{N}$, we give the following assumptions:

(F3) $f(x, u) u \geq 4 F(x, u) \geq 0$ for all $u \in R$ and a.e. $x \in R^{N}$.

(F4) $\frac{F(x, u)}{|u|^{4}} \rightarrow \infty$ as $|u| \rightarrow \infty$ uniformly in $x \in R^{N}$.

We have our main result as follow.

Theorem 1.1. Suppose that $(V),(F),(F 1)-(F 4)$ are satisfied. Then (1.1) has a 
ground state solution, there is a nontrivial critical point of $I$, such that $I(u)=\inf _{N} I$.

Our approach is the same a new linking-type result of [21] involving the $\mathrm{Ne}$ hari-Pankov manifold. In the next section, we present a critical point theory and variational setting. In Section 3, we state some relevant lemmas and prove Theorem 1.1.

\section{Preliminaries and Variational Setting}

Set Hilbertspace

$$
H^{1}\left(R^{N}\right)=\left\{u \in L^{2}\left(R^{N}\right), \nabla u \in L^{2}\left(R^{N}\right)\right\}
$$

with the norm

$$
\|u\|_{H^{1}}=\left(\int_{R^{N}}\left(|\nabla u|^{2}+u^{2}\right) \mathrm{d} x\right)^{\frac{1}{2}}
$$

To prove our theorems, let

$$
X=\left\{u \in H^{1}\left(R^{N}\right) \mid \int_{R^{N}}\left(a|\nabla u|^{2}+V(x) u^{2}\right) \mathrm{d} x<\infty\right\}
$$

and define the inner product of $X$ by the following formula:

$$
\langle u, v\rangle=\int_{R^{N}} a\left\langle\nabla u^{+}, \nabla u^{+}\right\rangle+V(x)\left\langle u^{+}, u^{+}\right\rangle \mathrm{d} x-\int_{R^{N}} a\left\langle\nabla u^{-}, \nabla u^{-}\right\rangle+V(x)\left\langle u^{-}, u^{-}\right\rangle \mathrm{d} x
$$

and norm given by $\|u\|=\langle u, u\rangle^{\frac{1}{2}}$, where $u=u^{+}+u^{-}, v=v^{+}+v^{-} \in X=X^{+} \oplus X^{-}$, therefore $X$ is a Hilbert space with the norm \|\| . It is easy to see that

$$
\int_{R^{N}}\left(a|\nabla u|^{2}+V(x) u^{2}\right) \mathrm{d} x=\left\|u^{+}\right\|^{2}-\left\|u^{-}\right\|^{2} .
$$

Let $X^{-}$is the finite dimentional space spanned by the negative eigenfunctions with $\lambda_{i}<0,(i=1, \cdots, n)$ and let $X^{+}=\left(X^{-}\right)^{\perp}$. In view of $(V)$, we may find continuous projections $u^{+}$and $u^{-}$of $X$ onto $X^{+}$and $X^{-}$, respectively, such that $X=X^{+}+X^{-}$and $X^{+}$is the positive eigenspace and $X^{-}$is the negative eigenspace of the operator $-a \Delta+V$.

For any $t \in\left[2,2^{*}\right]$, the embedding $x \rightarrow L^{t}\left(R^{N}\right)$ is continuous. Consequently, there is a constant $\gamma_{t}>0$, such that

$$
\|u\|_{t} \leq \gamma_{t}\|u\|, \forall u \in X
$$

Moreover, we know that under assumption $(V)$, the embedding $x \rightarrow L^{t}\left(R^{N}\right)$ is compact for any $t \in\left[2,2^{*}\right)$ by lemma 3.4 in [22].

Then the relative functional of (1.1) can be writed by

$$
I(u)=\frac{1}{2}\left\|u^{+}\right\|^{2}-\frac{1}{2}\left\|u^{-}\right\|^{2}+\frac{1}{4}\|u\|_{2}^{4}-\int_{R^{N}} F(x, u) \mathrm{d} x
$$

and under assumptions $(F 1)$ and $(F 2), I \in C^{1}(X, R)$, for any $u, v \in X$,

$$
\left\langle I^{\prime}(u), v\right\rangle=\left(a+b \int_{R^{N}}|\nabla u|^{2} \mathrm{~d} x\right) \int_{R^{N}} \nabla u \nabla v \mathrm{~d} x+\int_{R^{N}} V(x) u v \mathrm{~d} x-\int_{R^{N}} f(x, u) v \mathrm{~d} x .
$$

From the assumptions that $(F 1)$ and $(F 2)$, we know a functional $I \in C^{1}(X, R)$, 
then the following conditions hold:

(A1) $I$ is lower semicontinuous.

(A2) $I^{\prime}$ is weak continuous.

Let $P \subset X \backslash X^{-}$and $P \neq \varnothing$. The linking geometry of $I$ is described by the following conditions:

(A3) There exists $r>0$, such that $\inf _{u \in X^{+},\|u\|=r} I(u)>0$.

(A4) For every $u \in P$, there exists $R(u)>r$, such that $\sup _{\partial M(u)} I \leq I(0)=0$

where $M(u):=\left\{t u+v \in X \mid t \geq 0, v \in X^{-}\right.$and $\left.\|t u+v\| \leq R(u)\right\}$.

(A5) If $u \in N$, then $I(u) \geq I(t u+v)$ for any $t \geq 0$ and $v \in X^{-}$where $N=\left\{u \in P \mid\left\langle I^{\prime}(u), u\right\rangle=0\right.$ and $\left\langle I^{\prime}(u), v\right\rangle=0$ for any $\left.v \in X^{-}\right\}$.

If $P=X \backslash X^{-}$, then $N$ has been introduced by Pankov [23].

For any $A \subset X, I \subset[0, \infty)$, such that $0 \in I$, and $h: A \times I \rightarrow X$, we collect the following assumptions [24]:

(h1) $h$ is a continuous.

(h2) $h(0, u)=u$ for all $u \in A$.

(h3) $I(u) \geq I(h(u, t))$ for all $(u, t) \in A \times I$.

(h4) each $(u, t) \in A \times I$ has an open neighborhood $W$ in the product topology of $(X, T)$ and $I$ such that the set $\{v-h(v, s):(v, s) \in W \cap(A \times I)\}$ is contained in a finite dimentional subspace of $X$.

Theorem 2.1. (linking theory [21] [24]) Suppose that $I \in C^{1}(X, R)$ satisfies conditions A1-A4).

Then there exists a Cerami sequence $\left\{u_{n}\right\}$ (i.e. $I\left(u_{n}\right) \rightarrow c$, $\left.\left(1+\left\|u_{n}\right\|\right) I^{\prime}\left(u_{n}\right) \rightarrow\right)$, where

$$
\begin{gathered}
c=\inf _{u \in P} \inf _{h \in \Gamma(u)} \sup _{u^{\prime} \in M(u)} I\left(h\left(u^{\prime}, 1\right)\right)>0 \\
\Gamma(u)=\{h \in C(M(u) \times[0,1]) \mid h \text { satisfies (h1) }-(\mathrm{h} 4)\} .
\end{gathered}
$$

Suppose that in addition (A5) hold. Then $c \leq \inf _{N} I$, and if $c \geq I(u)$, for some critical point $u \in P$. then

$$
c=\inf _{N} I
$$

where $N=\left\{u \in P \mid\left\langle I^{\prime}(u), u\right\rangle=0\right.$ and $\left\langle I^{\prime}(u), v\right\rangle=0$ for any $\left.v \in X^{-}\right\}$.

\section{Proof of Theorem}

We need the following lemmas.

Lemma 3.1. Assume that $I \in C^{1}(X, R)$ and assumptions $(V),(F),(F 1)$ and $(F 2)$ are satisfied, then conditions $(A 1)$ and $(A 2)$ are hold.

Proof. Set $J(u)=\int_{R^{N}} F(x, u) \mathrm{d} x$. According to (1.5), it suffices to show that $J(u)$ is weakly continuous on $X$.

For any $\varepsilon>0$, by $(F 1)$ and $(F 2)$, there is $C_{\varepsilon}>0$, such that

$$
|f(x, u)|=\varepsilon|u|+C_{\varepsilon}|u|^{p-1} \text { for } u \in R
$$

let $\left\{u_{n}\right\} \subset X$ and $u_{n} \rightarrow u$ in $X$, then $\left\{u_{n}\right\}$ is bounded in $X$ and converges to 
$u$ in $L^{t}\left(R^{N}\right)$, where $2 \leq t<2^{*}$, by (3.1) we have

$$
\begin{aligned}
\left|J\left(u_{n}\right)-J(u)\right| & =\left|\int_{R^{N}} F\left(x, u_{n}(x)\right)-F(x, u(x)) \mathrm{d} x\right| \\
& =\left|\int_{R^{N}} \int_{0}^{1} f\left(x, u+s\left(u_{n}-u\right)\right)\left(u_{n}-u\right) \mathrm{d} s \mathrm{~d} x\right| \\
& \leq \int_{R^{N}}\left[\varepsilon\left|u+s\left(u_{n}-u\right)\right|+C_{\varepsilon}\left|u+s\left(u_{n}-u\right)\right|^{p-1}\right]\left|u_{n}-u\right| \mathrm{d} x \\
& \leq \int_{R^{N}}\left[\varepsilon\left(|u|+\frac{1}{2}\left|u_{n}-u\right|\right)+C_{\varepsilon, p}\left(|u|^{p-1}+\left|u_{n}-u\right|^{p-1}\right)\right]\left|u_{n}-u\right| \mathrm{d} x \\
& \leq c\left(\varepsilon+C_{\varepsilon, p}\left\|u_{n}-u\right\|_{p}\right) \leq C_{\varepsilon, n}
\end{aligned}
$$

Therefore, $J(u)$ is weakly continuous on $X$. This shows $(A 1)$ and $(A 2)$ hold.

Lemma 3.2. Assume that assumptions $(F),(F 1)$ and $(F 2)$ are satisfied, then condition $(A 3)$ are hold.

Proof. For any $0<\varepsilon<\frac{1}{4 \gamma_{2}^{2}}\left(\gamma_{2}\right.$ is depended on (2.1)), by (3.1) we know that there is $C_{\varepsilon}>0$ such that

$$
|F(x, u)| \leq \frac{\varepsilon}{2}|u|^{2}+\frac{C_{\varepsilon}}{p}|u|^{p} \text { for } u \in R .
$$

Note that (F4) implies $p>4$, then there is $r>0$, set $S=\left\{u \in X^{+},\|u\|=r\right\}$, for every $u \in S$, from (3.2) we have

$$
\begin{aligned}
I(u) & =\frac{1}{2}\left\|u^{+}\right\|+\frac{1}{4}\|u\|_{2}^{4}-\int_{R^{N}} F(x, u) \mathrm{d} x \\
& \geq \frac{1}{2}\left\|u^{+}\right\|^{2}-\frac{\varepsilon}{2}\|u\|_{2}^{2}-\frac{C_{\varepsilon}}{p}\|u\|_{p}^{p} \\
& \geq \frac{1}{4}\left(1-\gamma_{2}^{2} \varepsilon\right)\left\|u^{+}\right\|^{2}
\end{aligned}
$$

Therefore

$$
\inf _{u \in s} I(u) \geq \frac{1}{4}\left(1-\gamma_{2}^{2} \varepsilon\right) r^{2}>0
$$

This shows $(A 3)$ hold.

Hence similarly as in [17] [19], we obtain that conditions $(A 1)-(A 3)$ are hold. Moreover, obviously, $I(0)=0, I$ has the linking geometry.

Lemma 3.3. Assume that assumptions $(V),(F),(F 1)-(F 4)$ are satisfied, then condition (A4) is hold.

Proof. Choose a fixed $u \in P$ and there are $t_{n}>0$ and $v_{n} \in X^{-}$such that $I\left(t_{n} u+v_{n}\right)>0$ and $\left\|t_{n} u+v_{n}\right\| \rightarrow \infty$ as $n \rightarrow \infty$, let $z_{n}=\frac{t_{n} u+v_{n}}{\left\|t_{n} u+v_{n}\right\|}$ and we let $z_{n} \rightarrow z$ in $X$ and $z_{n}(x) \rightarrow z(x)$ a.e. $R^{N}$ for some $z \in X$. Since

$$
0<I\left(t_{n} u+v_{n}\right) \leq \frac{1}{2}\left\|t_{n} u^{+}\right\|^{2}-\frac{1}{2}\left\|t_{n} u^{-}+v_{n}\right\|^{2}+\frac{1}{4}\left\|t_{n} u^{+}\right\|^{4}
$$

then

$$
0<\frac{t_{n}^{4}}{\left\|t_{n} u+v_{n}\right\|^{4}}\left\|u^{+}\right\|^{4}=\left\|z_{n}^{+}\right\|^{4} \leq\left\|z_{n}\right\|^{4}=1
$$


and we may assume that $z^{+} \neq 0$. Hence $z \neq 0$ and

$$
\left|t_{n} u(x)+v_{n}(x)\right|=\left|z_{n}(x)\right|\left\|t_{n} u+v_{n}\right\| \rightarrow \infty \text { as } n \rightarrow \infty \text { and } z(x) \neq 0 .
$$

Then by (F4) and Fatou's lemma, we have

$$
0<\frac{I\left(t_{n} u+v_{n}\right)}{\left\|t_{n} u+v_{n}\right\|^{4}} \leq \frac{\gamma_{2}^{4}}{4}-\int_{R^{N}} \frac{F\left(x, t_{n} u+v_{n}\right)}{\left|t_{n} u+v_{n}\right|^{4}}\left|z_{n}\right|^{4} \mathrm{~d} x \rightarrow-\infty \text { as } t_{n} \rightarrow \infty
$$

and is a contradiction. This shows $(A 4)$ hold.

Remark 3.4. The inspection of proof of lemma 3.3, then due to conclusion of that lemma 3.3, for any $t \geq 0, v \in X^{-}$such that $(A 5)$ holds.

Lemma 3.5. Assume that $(V),(F),(F 1)$ and $(F 2)$ are satisfied. If $\left\{u_{n}\right\} \in X$ is a bounded Cerami sequence in $X$, then $\left\{u_{n}\right\}$ has a convergent subsequence.

Proof: Let $\left\{u_{n}\right\}$ is a Cerami sequence in $X$, that is $I\left(u_{n}\right) \rightarrow c$ and $\left(1+\left\|u_{n}\right\|\right) I^{\prime}\left(u_{n}\right) \rightarrow 0$.

From $I\left(u_{n}\right) \rightarrow \infty$ as $\left\|u_{n}\right\| \rightarrow \infty$, we get that $I$ is coercive.

Hence it is easy to get that $\left\{u_{n}\right\}$ is bounded. Since

$$
o\left(\left\|u_{n}\right\|\right)=\left\langle I^{\prime}\left(u_{n}\right), u_{n}\right\rangle=\left\|u_{n}^{+}\right\|^{2}-\left\|u_{n}^{-}\right\|^{2}+\left\|u_{n}\right\|_{2}^{4}-\int_{R^{N}} f\left(x, u_{n}\right) u_{n} \mathrm{~d} x
$$

One has

$$
o(1)+\left\|u_{n}^{-}\right\|^{2}=\left\|u_{n}^{+}\right\|^{2}+\left\|u_{n}\right\|_{2}^{4}-\int_{R^{N}} f\left(x, u_{n}\right) u_{n} \mathrm{~d} x
$$

Passing to a subsequence, we may assume that $u_{n} \rightarrow u$ in $X$. Therefore

$$
0=\left\langle I^{\prime}(u), u\right\rangle=\left\|u^{+}\right\|^{2}-\left\|u^{-}\right\|^{2}+\|u\|_{2}^{4}-\int_{R^{N}} f(x, u) u \mathrm{~d} x
$$

and we have

$$
\left\|u^{-}\right\|^{2}=\left\|u^{+}\right\|^{2}+\|u\|_{2}^{4}-\int_{R^{N}} f(x, u) u \mathrm{~d} x
$$

Moreover, since $\operatorname{dim} X^{-}<\infty$, we have that $u_{n}^{-} \rightarrow u^{-}$. Then combine (3.3) with (3.4) push out

$$
\lim _{n \rightarrow \infty}\left(\left\|u_{n}^{+}\right\|^{2}+\left\|u_{n}\right\|_{2}^{4}-\int_{R^{N}} f\left(x, u_{n}\right) u_{n} \mathrm{~d} x\right)=\left\|u^{+}\right\|^{2}+\|u\|_{2}^{4}-\int_{R^{N}} f(x, u) u \mathrm{~d} x
$$

It follows from $(A 2)$ and Fatou's lemma that

$$
\varliminf_{n \rightarrow \infty}\left(\left\|u_{n}\right\|_{2}^{4}-\int_{R^{N}} f\left(x, u_{n}\right) u_{n} \mathrm{~d} x\right) \geq\|u\|_{2}^{4}-\int_{R^{N}} f(x, u) u \mathrm{~d} x
$$

Combining (3.5) and (3.6), we can get

$$
\underline{\lim }_{n \rightarrow \infty}\left\|u_{n}^{+}\right\|^{2} \leq\left\|u^{+}\right\|^{2}
$$

and then $\left\|u_{n}^{+}\right\| \rightarrow\left\|u^{+}\right\|$. Consequently, $u_{n} \rightarrow u$ in $X$.

This shows lemma's conclusion hold.

Proof of Theorem 1.1. Observe that (F3) implies that $P=X \backslash X^{-}$. In view of theorem 2.1, there is a bounded Cerami sequence $\left\{u_{n}\right\}$ and by lemma 3.5 , $\left\{u_{n}\right\}$ has a convergent subsequence. Then passing to a subsequence, let $u_{n} \rightarrow u$ in $X$, then $u_{n}(x) \rightarrow u(x)$ a.e. in $R^{N}$. From lemma 3.1, we can obtain that $I$ is lower semicontinuous. One has that 


$$
\int_{R^{N}}|\nabla u|^{2} \mathrm{~d} x \leq \varliminf_{n \rightarrow \infty} \int_{R^{N}}\left|\nabla u_{n}\right|^{2} \mathrm{~d} x
$$

Observe that by $(F 3)$ and in view of Fatou's lemma that

$$
c=\varliminf_{n \rightarrow \infty} I\left(u_{n}\right)=\varliminf_{n \rightarrow \infty}\left(I\left(u_{n}\right)-\frac{1}{4}\left\langle I^{\prime}\left(u_{n}\right), u_{n}\right\rangle\right) \geq I(u)
$$

Since $u \in N$, then by theorem 2.1 we have

$$
c=\inf _{N} I=I(u)
$$

This shows the main result hold.

\section{Conclusion}

To sum up the above arguments, we through the linking theory to prove the existence of ground state solution of Schrödinger-Kirchhoff type equation.

\section{Conflicts of Interest}

The author declares no conflicts of interest regarding the publication of this paper.

\section{References}

[1] Brezis, H. and Lieb, E. (1984) Minimum Action Solutions of Some Vector Field Equations. Communications in Mathematical Physics, 96, 97-113. https://doi.org/10.1007/BF01217349

[2] Coti Zelati, V. and Rabinowitz, P.H. (1992) Homoclinic Type Solutions for a Semilinear Elliptic PDE on $R^{\mathrm{n}}$. Communications on Pure and Applied Mathematics, 45, 1217-1269. https://doi.org/10.1002/cpa.3160451002

[3] Troestler, C. and Willem, M. (1996) Nontrivial Solution of a Semilinear Schrodinger Equation. Communications in Partial Differential Equations, 21, 1431-1449. https://doi.org/10.1080/03605309608821233

[4] Kryszewski, W. and Szulkin, A. (1998) Generalized Linking Theorem with an Application to a Semilinear Schrodinger Equation. Advances in Differential Equations, 3, 441-472.

[5] Kirchho, G. (1883) Mechanik, Teubner, Leipzig.

[6] Arosio, A. and Panizzi, S. (1996) On the Well-Posedness of the Kirchhoff String. Transactions of the American Mathematical Society, 348, 305-330. https://doi.org/10.1090/S0002-9947-96-01532-2

[7] D'Ancona, P. and Spagnolo, S. (1992) Global Solvability for the Degenerate Kirchhoff Equation with Real Analytic Data. Inventiones Mathematicae, 108, 247-262. https://doi.org/10.1007/BF02100605

[8] Lions, J.L. (1978) On Some Questions in Boundary Value Problems of Mathematical Physics. North-Holland Mathematics Studies, 30, 284-346. https://doi.org/10.1016/S0304-0208(08)70870-3

[9] Ma, T.F. and Munoz Rivera, J.E. (2003) Positive Solutions for a Nonlinear Nonlocal Elliptic Transmission Problem. Applied Mathematics Letters, 16, 243-248. https://doi.org/10.1016/S0893-9659(03)80038-1

[10] Cavalcanti, M.M., Domingos Cavalcanti, V.N. and Soriano, J.A. (2001) Global Existence and Uniform Decay Rates for the Kirchhoff-Carrier Equation with Nonlinear 
Dissipation. Advances in Difference Equations, 6, 701-730.

[11] Perera, K. and Zhang, Z. (2006) Nontrival Solutions of Kirchhoff-Type Problems via the Yang Index. The Journal of Differential Equations, 221, 246-255. https://doi.org/10.1016/j.jde.2005.03.006

[12] Wu, X. (2011) Existence of Nontrivial Solutions and High Energy Solutions for Schrodinger-Kirchhoff-Type Equations in RN. Nonlinear Analysis, 12, 1278-1287. https://doi.org/10.1016/j.nonrwa.2010.09.023

[13] He, X. and Zou, W. (2009) Infinitely Many Positive Solutions for Kirchhoff-Type Problems. Nonlinear Analysis. Theory, Methods \& Applications, 70, 1407-1414. https://doi.org/10.1016/j.na.2008.02.021

[14] He, X. and Zou, W. (2014) Ground States for Nonlinear Kirchhoff Equations with Critical Growth. Annali di Matematica Pura ed Applicata, 193, 473-500. https://doi.org/10.1007/s10231-012-0286-6

[15] Chen, S. and Liu, S. (2014) Standing Waves for 4-Superlinear Schrodinger-Kirchhoff Equations. Mathematical Methods in the Applied Sciences, 38, 1-9. https://doi.org/10.1002/mma.3212

[16] Li, Q. and Wu, X. (2014) A New Result on High Energy Solutions for Schrodinger-Kirchhoff Type Equations in $R^{\mathrm{N}}$. Applied Mathematics Letters, 30, 24-27. https://doi.org/10.1016/j.aml.2013.12.002

[17] Jin, J. and $\mathrm{Wu}, \mathrm{X}$. (2010) Infinitely Many Radial Solutions for Kirchhoff-Type Problems in $R^{\mathrm{N}}$. Journal of Mathematical Analysis and Applications, 369, 564-574.

[18] Sun, J. and Liu, S. (2012) Nontrivial Solutions of Kirchhoff Type Problems. Applied Mathematics Letters, 25, 500-504. https://doi.org/10.1016/j.aml.2011.09.045

[19] Wu, Y. and Liu, S. (2015) Existence and Multiplicity of Solutions for Asymptotically Linear Schrödinger-Kirchhoff Equations. Nonlinear Analysis. Theory, Methods \& Applications, 26, 1991-1998. https://doi.org/10.1016/j.nonrwa.2015.05.010

[20] Reed, M. and Simon, B. (1978) Methods of Modern Mathematical Physics. IV. Analysis of Operators. Academic Press, New York, London.

[21] Mederski, J. (2015) Ground States of a System of Nonlinear Schrödinger Equations with Periodic Potentials. Communications in Partial Differential Equations, 41, 1426-1440. https://doi.org/10.1080/03605302.2016.1209520

[22] Zou, W.M. and Schechter, M. (2006) Critical Point Theory and Its Applications. Springer, New York.

[23] Szulkin, A. and Weth, T. (2009) Ground State Solutions for Some Indefinite Variational Problems. Journal of Functional Analysis, 257, 3802-3822. https://doi.org/10.1016/j.jfa.2009.09.013

[24] Willem, M. (1996) Minimax Theorem. Birkhäuser Boston, Inc., Boston, MA. https://doi.org/10.1007/978-1-4612-4146-1 